\title{
Investigation of the Utility of Web-Based Videos about Varicose Veins
}

\author{
Zhoupeng $\mathrm{Wu}^{1}$ and Yukui $\mathrm{Ma}^{1, *}$ \\ 1 Department of Vascular Surgery, West China Hospital, Sichuan Province, China
}

* Corresponding author: Yukui Ma, Department of Vascular Surgery, West China Hospital, Sichuan Province, China. Email: hxyymyk@126.com

Received 2021 January 05; Revised 2021 January 27; Accepted 2021 February 26

\begin{abstract}
Background: Very few investigations and studies have been conducted on the provided information about varicose veins in web-based media worldwide.

Objectives: The present study aimed to investigate the categorization, characteristics, scientificity, and accuracy of such videos uploaded to YouTube.

Methods: YouTube was searched during November 2019, and the search keywords were "varicose vein", "varicose veins", and "varicose veins of lower extremities". The videos collected by retrieval were classified as useful, partially useful, and completely useless based on their scientific content, contemporariness, and accuracy. The analysis was mainly performed on the data collected from videos that were categorized as useful.

Results: In total, 635 (75.6\%) out of 840 collected videos were excluded. Most videos on the website were uploaded by medical staff $(31 \%, n=64)$. Based on the results, among the videos included in the data analysis, videos uploaded by official agencies were viewed more times than those uploaded by others. In addition, most of the useful videos were uploaded by official institutions.

Conclusion: Official institutions should provide more web-based videos with up-to-date, complete, and accurate information about varicose veins. Furthermore, effective search tools are needed for the identification of the videos uploaded by academic sources and institutions.

Keywords: Useful, Varicose veins, Web
\end{abstract}

\section{Background}

Based on previous studies, one-fifth of the world population has varicose veins, and its incidence rate is higher in the elderly, compared to others. Primary varicose veins of lower extremities are very common in developed and developing countries (1). Treatment options for this disease include pressure treatment with elastic stockings, thermal ablation techniques, sclerotherapy, and traditional open surgery. Despite the fact that traditional open surgery is still used, other methods, such as radiofrequency ablation (RFA), intraluminal laser therapy (EVLA), and foam sclerotherapy, are becoming more and more common $(2,3)$.

This disease causes a large social cost and a reduction in the labor force in society (4). With the gradual development of information technology platforms in China, increasing numbers of people are obtaining information from the internet, especially regarding education, medical care, and lifestyle. According to the published literature, approximately 8 out of every 10 people search for medical-related information online. Moreover, video data is ranked as the first choice for users due to its various features. YouTube is the largest video hosting platform in the world, with a daily broadcast volume of 1.18 billion, covering most of the population of China.

Few studies have investigated videos related to varicose veins in web-based media in the world (5).

\section{Objectives}

The present study aimed to investigate the quality of information on varicose veins available on YouTube in order to provide reasonable suggestions for the improvement of the public understanding and knowledge about this disease.

\section{Methods}

For the purposes of the study, YouTube was searched during November 2019 using the keywords "varicose vein", "varicose veins", and "varicose veins of lower extremities". The most recent 205 videos were investigated for the purposes of the study. In total, 840 videos were found and evaluated. The exclusion criteria were duplications, irrelevant content, advertisements, alternative medicine topics, and non-Chinese/ English language videos.

In total, 205 videos were selected for further evaluation. The main investigated parameters included the video characteristics, content, number of views, occupation of the person who uploaded the video, duration of the video, and whether it contained animation or not. Two vascular surgeons watched each included video at the same time to assess their effect on the general public. If the two vascular

Copyright (c) 2021, Author(s). This is an open-access article distributed under the terms of the Creative Commons Attribution-NonCommercial 4.0 International License (http://creativecommons.org/licenses/by-nc/4.0/) which permits copy and redistribute the material just in noncommercial usages, provided the original work is properly cited 
surgeons disagreed on the video, an independent third-party person evaluated the video and made a final decision.

The content of the videos was divided into three groups, namely useful, partially useful, or useless, according to their scientific content (i.e., general information about the disease, diagnosis, and treatment), contemporariness, and accuracy, with emphasis on data insight and representation of visual features. Videos that met all the conditions were defined as useful videos. Videos that were inadequate or met only some of the criteria were defined as partially useful, and the remaining were defined as useless. Statistical analysis was performed only on the videos that were categorized as useful.

\subsection{Statistical analysis}

Frequencies and percentages were used to represent univariate categorical variables, and means and standard deviations were used to represent interval variables. In addition, an independent student t-test was used to test the differences between interval variables.

When it was assumed that the ordinal categories in the variables represented a basic normal distribution, Pearson product-moment correlation and multiple regression analysis were used to analyze the data. Moreover, the chi-squared test and Kendall's rank test were used for ordinal variables. Finally, all results were analyzed using SPSS software (version 18.0). It should be noted that a pvalue of less than 0.05 was considered statistically significant.

\section{Results}

In total, $635(75.6 \%)$ out of the 840 selected videos were excluded, and more than half of the videos were played repeatedly. Table 1 summarizes the exclusion criteria, and Table 2 tabulates the characteristics of the 205 videos that were included in the study. Many of the uploaded videos were produced by healthcare professionals ( $n=72,33.0 \%)$. Furthermore, most of the videos contained treatment-related content, such as RFA, EVLA, traditional open surgery, and sclerotherapy $(n=174$, $84.9 \%$ ), as shown in Table 2. No information regarding surgical complications was found in the videos.

In terms of the sources of the videos, videos uploaded by official research institutes/associations

\begin{tabular}{lc}
\hline Table 1. Exclusion criteria & \\
\hline & $\mathbf{N}(\%)$ \\
\hline Duplicate videos & $374(58.9)$ \\
Irrelevant content & $54(8.5)$ \\
Advertisements & $138(21.7)$ \\
Alternative medicine topics & $45(7.1)$ \\
Non-Chinese/English language videos & $24(3.8)$ \\
Total & 635 \\
\hline
\end{tabular}

\begin{tabular}{|c|c|c|}
\hline Date uploaded & $\mathbf{n}$ & $\%$ \\
\hline 2015 or earlier & 68 & 33.2 \\
\hline 2016 & 45 & 22.0 \\
\hline 2017 & 44 & 21.5 \\
\hline 2018 & 48 & 23.3 \\
\hline \multicolumn{3}{|l|}{ Uploaded by individual or institution } \\
\hline Healthcare professional (i.e., physician, nurse) & 72 & 35.1 \\
\hline Hospital & 54 & 26.3 \\
\hline Television show & 26 & 12.7 \\
\hline General public & 20 & 9.8 \\
\hline Network media & 21 & 10.2 \\
\hline Official institution/associations & 12 & 5.9 \\
\hline $\begin{array}{l}\text { Provision of general information on the } \\
\text { disease }\end{array}$ & 163 & 79.5 \\
\hline $\begin{array}{l}\text { Provision of general information on the } \\
\text { diagnosis }\end{array}$ & 42 & 20.5 \\
\hline $\begin{array}{l}\text { Provision of general information on the } \\
\text { treatment }\end{array}$ & 174 & 84.9 \\
\hline \multicolumn{3}{|l|}{ Complete treatment } \\
\hline EVLA, RFA & 97 & 47.3 \\
\hline Sclerotherapy & 65 & 31.7 \\
\hline Compression & 32 & 15.6 \\
\hline Open surgery & 54 & 26.3 \\
\hline Other treatment & 9 & 4.3 \\
\hline Presence of animation in the video & 32 & 15.6 \\
\hline Total & 205 & 100 \\
\hline
\end{tabular}

had significantly more views than the others. Views of the videos uploaded by other sources did not differ significantly from each other. Furthermore, a significantly higher number of videos uploaded by official agencies/associations were useful, compared to those uploaded by others. No significant associations were found between the other sources of uploads and the number of useful videos. It should also be mentioned THAT The videos categorized as useful had significantly more views compared to the others (Table 3).

\section{Discussion}

With the gradual development of information technology networks in China, high-speed internet has become accessible to the general public. In this regard, videos are known as sources of information, especially in the field of medical education. The present study mainly aimed to analyze and evaluate the quality of videos on varicose veins present on the largest video hosting platform in the world, YouTube.

Characteristics of videos on varicose veins uploaded to YouTube were investigated, and most of them $(61.4 \%)$ were found to be uploaded by healthcare professionals or hospitals. However, these videos did not have more views or usefulness, compared to the other ones.

Studies on web-based videos on other diseases have shown that videos uploaded by academic institutions are more accurate, receive more attention, and are downloaded more than others $(6,7)$. Videos focusing on varicose veins can also be found on other websites, such as youku.com (8).

In total, 205 videos were included this study based on specific inclusion and exclusion criteria. It 


\begin{tabular}{lccc}
\hline \multicolumn{4}{l}{ Table 3. Distribution of video scores and download rates based on the upload source } \\
\\
\hline General public & 9432 & $875(214-10927)$ & 38.8 \\
Healthcare professional & 12237 & $968(356-4702)$ & 56.2 \\
Hospital & 13784 & $1556(318-8548)$ & 71.3 \\
Television show & 16178 & $1658(274-10549)$ & 89.6 \\
Network media & 27346 & $3095(467-27563)$ & 43.5 \\
Official institution/associations & 101276 & $9324(1906-72048)$ & 100 \\
\end{tabular}

IQR: interquartile distance

1) Based on the analysis, scores and download rates of videos uploaded by official institutions/associations were significantly different from those of other videos.

2) Based on the analysis, scores and download rates of videos uploaded by official institutions/associations or TV programs were significantly different from those of other videos.

should be mentioned that many videos on the website that deal with alternative medicine were excluded from this research. Ahadiat 0 et al. reported that many websites have promoted non-surgical treatments for varicose veins, including herbal medicines, and also found that majority of the videos are uploaded by hospitals (9). In this study, it was found that hospitals were the third most common creator of videos.

An increasing number of videos reflect the latest treatments for varicose veins (10). Treatment options for this disease include pressure treatment with elastic stockings, thermal ablation techniques, sclerotherapy, and traditional open surgery. Although traditional open surgery is still used, other methods such as RFA, EVLA, and foam sclerotherapy are becoming more and more popular (11). These videos provide a detailed description of the latest treatments for varicose veins; however, they do not explain the complications of the treatments. This is consistent with the results of our observations, and indicates an urgent need for improvement in this area.

YouTube has published a series of information sources that have not been peer-reviewed. We believe that patients cannot be expected to accurately distinguish poor-quality videos from good-quality ones. By the addition of new features, such as allowing users to search for videos citing references, this unregulated video library can be made more reliable. In addition, the use of new information dissemination methods (e.g., videos that may encourage users to report poor-quality information) may improve search accuracy and results.

Another possible way to solve this problem is for official organizations to produce or promote the production of high-quality information (including videos) on various diseases and treatments. Moreover, they can raise the awareness of doctors regarding these sources of information in a way that patients can be referred to them. However, even such measures cannot prevent the patients, especially in the early stages of the diagnosis and treatment process, from conduction of their own unguided searches.

Therefore, doctors will need to be updated on the latest trends in online health information in order to be able to provide consultations for patients appropriately. The main objective of this research was to make clinicians use online videos more (especially those uploaded to YouTube) as the information source currently used by patients in order to promote better discussions among clinicians, patients, and vascular experts. Besides, it also aimed to enhance the level of information of patients, and hope to eventually lead to a better understanding and consensus on the composition of patient education videos.

Since YouTube is the largest video website in the world and the scientific rigor and accuracy of its videos have not been strictly monitored, some of its content may lead to incorrect perceptions. To address issues of scientific rigor and accuracy, it may be feasible to validate specific videos on YouTube and create special annotations or links to official agencies or academic teams.

\subsection{Limitations}

Online media content is constantly changing with time. The present study used keywords to search YouTube, and only included the first two pages of the retrieved videos (14 videos/page).

\section{Conclusion}

It can be concluded that videos about varicose veins available on YouTube are not very useful. Official agencies and/or public associations should be encouraged to produce and upload comprehensive content about the disease to ensure scientificity and accuracy, including videos about the latest treatment options and potential treatment risks, complications, or adverse events. Finally, more additional search tools will be helpful in the identification of videos uploaded by government sources, official agencies, or academic sources.

\section{References}

1. Dharmarajah B, Lane TR, Moore HM, Neumann HM, Rabe E, Wittens $\mathrm{CH}$, et al. The future of phlebology in Europe. Phlebology. 2014;29(1 Suppl):181-5. doi: 10.1177/0268355514527046. [PubMed: 24843107].

2. Eberhardt RT, Raffetto JD. Chronic venous insufficiency. 
Circulation. 2014;130(4):333-46. doi: 10.1161/CIRCULATIO NAHA.113.006898. [PubMed: 25047584].

3. Wittens C, Davies AH, Bækgaard N, Broholm R, Cavezzi A, Chastanet $S$, et al. Editor's choice - management of chronic venous disease: clinical practice guidelines of the European society for vascular surgery (ESVS). Eur J Vasc Endovasc Surg. 2015;49(6):678-737. doi: 10.1016/j.ejvs.2015.02.007. [PubMed: 25920631].

4. Carroll C, Hummel S, Leaviss J, Ren S, Stevens JW, Cantrell A, et al. Systematic review, network meta-analysis and exploratory cost-effectiveness model of randomized trials of minimally invasive techniques versus surgery for varicose veins. $\mathrm{Br} J$ Surg. 2014;101(9):1040-52. doi: 10.1002/bjs.9566. [PubMed: 24964976].

5. Bozoglan O, Mese B, Eroglu E, Ekerbiçer HC, Yasim A. Comparison of endovenous laser and radiofrequency ablation in treating varices in the same patient. J Lasers Med Sci. 2017;8(1):13-6. doi: 10.15171/jlms.2017.03. [PubMed: 28912938].

6. Yaylaci S, Serinken M, Eken C, Karcioglu O, Yilmaz A, Elicabuk $\mathrm{H}$, et al. Are YouTube videos accurate and reliable on basic life support and cardiopulmonary resuscitation? Emerg Med Australas. 2014;26(5):474-7. doi: 10.1111/1742-6723.12274. [PubMed: 25168312]
7. Mese B, Bozoglan O, Eroglu E, Erdem K, Acipayam M, Ekerbicer $\mathrm{HC}$, et al. A comparison of 1470-nm endovenous laser ablation and radiofrequency ablation in the treatment ofgreat saphenous veins $10 \mathrm{~mm}$ or more in size. Ann Vasc Surg. 2015;29(7):1368-72. doi: 10.1016/j.avsg.2015.03.063.

8. Kumar N, Pandey A, Venkatraman A, Garg N. Are video sharing web sites a useful source of information on hypertension? J Am Soc Hypertens. 2014;8(7):481-90. doi: 10.1016/j.jash.2014. 05.001. [PubMed: 25064770].

9. Ahadiat O, Higgins S, Ly A, Nazemi A, Wysong A. Review of endovenous thermal ablation of the great saphenous vein: endovenous laser therapy versus radiofrequency ablation. Dermatol Surg. 2018;44(5):679-88. doi: 10.1097/DSS.0000 000000001478. [PubMed: 29462021].

10. Malskat WS, Giang J, De Maeseneer MG, Nijsten TE, van den Bos RR. Randomized clinical trial of $940-$ versus $1470-\mathrm{nm}$ endovenous laser ablation for great saphenous vein incompetence. Br J Surg. 2016;103(3):192-8. doi: 10.1002/ bjs.10035. [PubMed: 26661521].

11. Wozniak W, Mlosek RK, Ciostek P. Complications and 'failure of endovenous laser ablation and radiofrequency ablation procedures in patients with lower extremity varicose veins in a 5-year follow-up. Vasc Endovascular Surg. 2016;50(7):475-83. doi: 10.1177/1538574416671247. [PubMed: 27681171]. 